\title{
IMPORTÂNCIA DO DIAGNÓSTICO DA FRATURA SUBCONDRAL DA CABEÇA DO FÊMUR, SUAS DIFERENÇAS COM A NECROSE AVASCULAR E SEU TRATAMENTO
}

\author{
THE IMPORTANCE OF THE DIAGNOSIS OF SUBCHONDRAL \\ FRACTURE OF THE FEMORAL HEAD, HOW TO DIFFERENTIATE IT \\ FROM AVASCULAR NECROSIS AND HOW TO TREAT IT
}

Giancarlo Polesello', Denis Seguchi Sakai ${ }^{2}$, Nelson K. Ono ${ }^{3}$, Emerson K. Honda ${ }^{4}$, Rodrigo Pereira Guimarães ${ }^{5}$, Walter Ricioli Júnior'

\section{RESUMO}

A fratura subcondral da cabeça femoral é afecção pouco conhecida e, consequentemente, pouco diagnosticada. O quadro clínico de dor aguda ou insidiosa com piora progressiva na região inguinal, exacerbada com carga e com alívio em repouso, deve levar à suspeita diagnóstica. O erro comumente feito com o diagnóstico de necrose avascular da cabeça do fêmur pode induzir a indicação de procedimentos invasivos desnecessários no quadril. Com o advento da ressonância nuclear magnética, o diagnóstico dessa afecção pode ser realizado observando os diferentes padrões de edema medular na cabeça do fêmur, possibilitando o tratamento precoce correto.

Descritores - Osteonecrose/diagnóstico; Cabeça do fêmur/ anormalidades; Imagem por ressonância magnética/métodos; Fraturas espontâneas/diagnóstico; Fraturas do quadril/radiografia

\section{ABSTRACT}

Subchondral fracture of the femoral head is an uncommon and underdiagnosed affection. The abrupt or gradual onset of groin pain with functional disability on weight-bearing that improves with a resting period should alert the orthopedic surgeon to the possibility of this diagnosis. The differential diagnosis from osteonecrosis of the femoral head can be provided by assessing the different patterns of bone edema on MRI studies of the hip, thus avoiding unnecessary invasive operations on the hip.

Keywords - Osteonecrosis/diagnosis; Femur head/abnormalities; Magnetic resonance imaging/methods; Fractures, spontaneous/ diagnosis; Hip fractures/ radiography

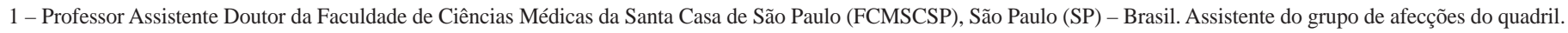
2 - Médico Residente do Departamento de Ortopedia e Traumatologia da Faculdade de Ciências Médicas da Santa Casa de São Paulo (FCMSCSP), São Paulo (SP) - Brasil.

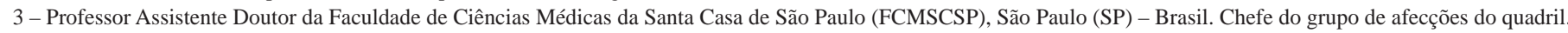

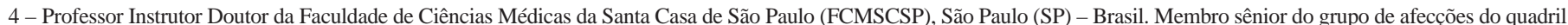

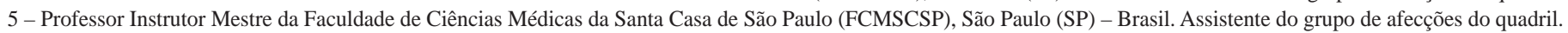

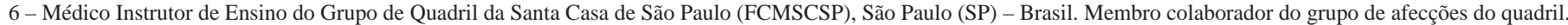

Agradecimento: A Denise Tokechi Amaral, Médica Radiologista do Medimagem do Hospital Beneficência Portuguesa de São Paulo

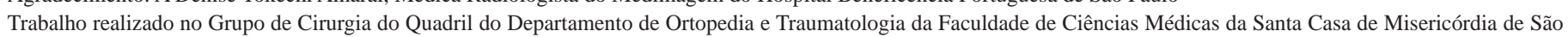
Paulo, "Pavilhão Fernandinho Simonsen” (DOT-FCMSCSP). Diretor Prof. Dr. Osmar Avanzi.

Correspondência: Pavilhão “Fernandinho Simonsen”, Rua Dr. Cesário Motta Jr., 112 - 01221-020, São Paulo, SP - Brasil. E-mail: giancarlopolesello@hotmail.com 


\section{INTRODUÇÃO}

Pelos muitos nomes encontrados na literatura para designar síndromes dolorosas do quadril, é de se prever que nenhum deles seja o adequado ou que todos sejam sinônimos.

Ainda não se sabe se a fratura subcondral, a osteoporose transitória, a desmineralização transitória, a osteólise transitória, a algodistrofia do quadril e a síndrome do edema medular são afecções diferentes e muitas dessas denominações são anteriores ao advento da RM.

A primeira descrição de osteoporose transitória foi realizada em 1959 em gestantes, tendo sido relatada melhora gradual após o parto num período de alguns meses $^{(1)}$, sendo posteriormente descrita em outras articulações e em homens de meia-idade com dor incapacitante que melhorava com alívio da carga de forma completa em seis a 12 meses $^{(2)}$.

Alguns autores sugerem que a osteoporose transitória é uma forma de distrofia simpático-reflexa do quadril ${ }^{(2)}$. Outros acreditam que a osteoporose transitória e a fratura subcondral são espectros diferentes da mesma doença; na fratura subcondral se observa a linha de fratura ${ }^{(3)}$. As fraturas subcondrais podem estar associadas a quadro de síndrome de edema medular transitório/osteoporose transitória ${ }^{(3-6)}$.

Em contrapartida, a necrose avascular da cabeça do fêmur e a fratura subcondral têm algumas características semelhantes, porém, suas evoluções e tratamentos são completamente diferentes, tornando fundamental seu correto diagnóstico.

A osteonecrose da cabeça femoral tem fisiopatologia incerta, cursando com congestão e edema medular precoces $^{(7)}$. Leva a incapacidade proporcional à dor, piorando progressivamente com carga e mesmo em repouso ${ }^{(8)}$. Dez por cento são de origem idiopática e $80 \%$ são causadas principalmente pelo excesso de álcool e uso de esteroides. O pico de incidência é entre a terceira e a quinta décadas de vida, não havendo predileção por sexo. Aproximadamente $50 \%$ dos casos acometem ambos os quadris ${ }^{(8)}$.

A osteoporose transitória é entidade incomum e autolimitada, variando de seis a 36 meses $^{(2,8-11)}$. Sua etiologia é desconhecida, sendo o único fator de risco conhecido a gestação ${ }^{(8)}$. Cursa com dor incapacitante sem antecedente de trauma, piorando com a deambulação e mobilização do quadril afetado ${ }^{(8,9)}$. Acomete três vezes mais homens de meia-idade do que mulheres e gestantes no terceiro trimestre. Na sua patogênese podem-se distinguir três fases temporais: inicial (duração de aproximadamente um mês), platô (duração de um a dois meses) e regressão (duração de aproximadamente quatro meses) $^{(8)}$.

\section{DIAGNÓSTICO}

O quadro clínico de dor aguda ou insidiosa, principalmente na virilha, mas também na nádega ou região anterior da $\operatorname{coxa}^{(9)}$, que piora com a deambulação e melhora com o alívio da carga, na ausência de fatores predisponentes para osteonecrose da cabeça femoral ou antecedente traumático em pacientes de meia-idade, deve alertar para a possibilidade do diagnóstico de fratura subcondral da cabeça do fêmur.

Os exames radiográficos na fase precoce da osteonecrose da cabeça femoral, na osteoporose transitória e na fratura subcondral não apresentam alteração, tornando necessário o estudo por ressonância magnética analisado por um radiologista experiente.

O diagnóstico de osteonecrose da cabeça femoral é feito pela $\mathrm{RM}^{(7,12)}$ e a área de osteonecrose caracterizase por formato em crescente, de aspecto geográfico, na região subcondral ântero-superior da cabeça femoral. É circundada por interface reativa com baixo sinal externo (esclerose) e alto sinal interno nas sequências ponderadas em $\mathrm{T} 2^{(13)}$. Essa área de hipersinal representa hiperemia e tecido de granulação na interface entre o osso viável e o desvitalizado (Figura 1), padrão de dupla linha que é diagnóstico definitivo de osteonecrose ${ }^{(14)}$ (Figura 2).

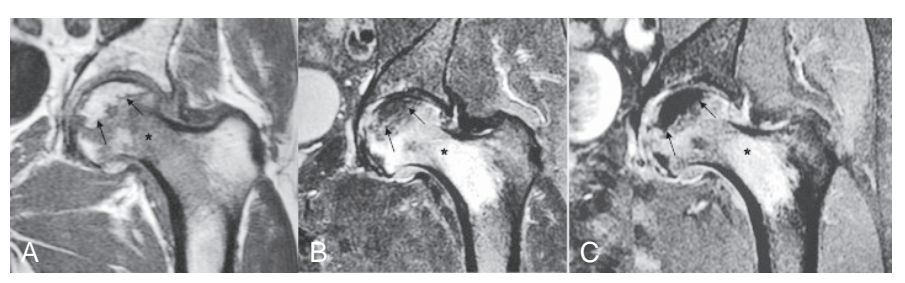

Figura 1 - Osteonecrose (seta) caracterizada por área com formato em crescente no plano coronal T1 (a). Na sequência T2 com saturação de gordura (b) é circundada pelo sinal de dupla linha (hipersinal central e hipossinal periférico). Na fase pós-contraste T1 com saturação de gordura (c), a área de necrose não apresenta realce, persistindo com baixíssimo sinal. $O$ edema da medular óssea $\left({ }^{*}\right)$ caracteriza-se por sinal intermediário em T1, hipersinal em T2 e na fase pós-contraste.

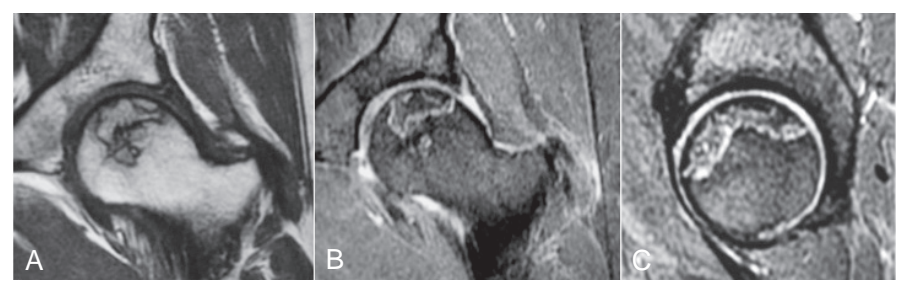

Figura 2 - Osteonecrose numa fase crônica ( ${ }^{*}$ ), caracterizada por área serpigiforme subcondral em toda a porção superior da cabeça femoral. Apresenta halo de esclerose no plano coronal T1 (a), sinal da dupla linha em T2 com saturação de gordura no plano coronal (b) e sagital (c) - hipersinal central (seta branca) e hipossinal periférico (seta preta). Não há sinais de edema da medular óssea nem colapso. 
A área de necrose inicialmente apresenta alto sinal em T1, mas posteriormente o sinal pode ser substituído por líquido ou esclerose ${ }^{(15)}$. O fator mais importante no prognóstico da osteonecrose é a estimativa da percentagem de superfície de carga comprometida ${ }^{(16)}$ (Figura 3).

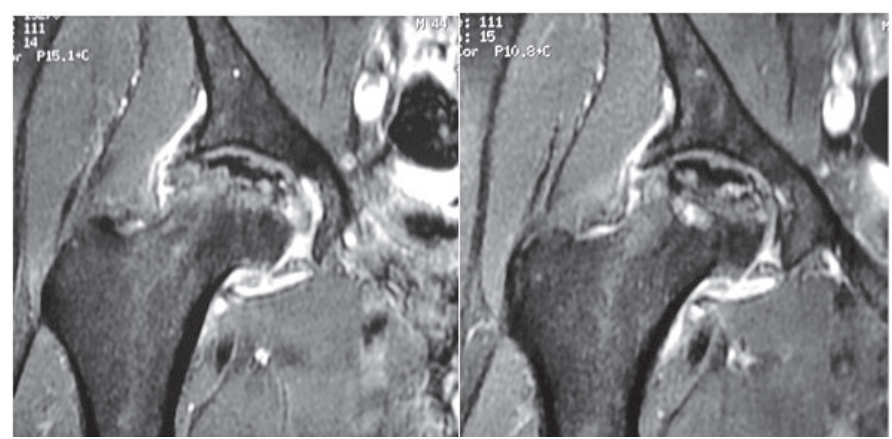

Figura 3 - Osteonecrose com colapso e artrose secundária. Plano coronal T1 pós-contraste com saturação de gordura. A área de necrose $\left({ }^{*}\right)$ apresenta baixíssimo sinal, pelo fato de não apresentar realce. Há perda da esfericidade (setas brancas), extensa reação osteofitária marginal tanto no acetábulo quanto na cabeça femoral, associada a derrame articular e sinovile (seta preta).

Na osteoporose transitória a ressonância nuclear magnética é o exame de escolha para o diagnóstico precoce ${ }^{(8,11,12)}$, mostrando hipossinal em T1 e hipersinal em T2 na cabeça e colo do fêmur e, ocasionalmente, no acetábulo ${ }^{(9,10,12,17)}$ (Figuras 4, 5 e 6). Diferentemente da osteonecrose, não ocorrem erosão óssea nem colapso subcondral (Figura 7).

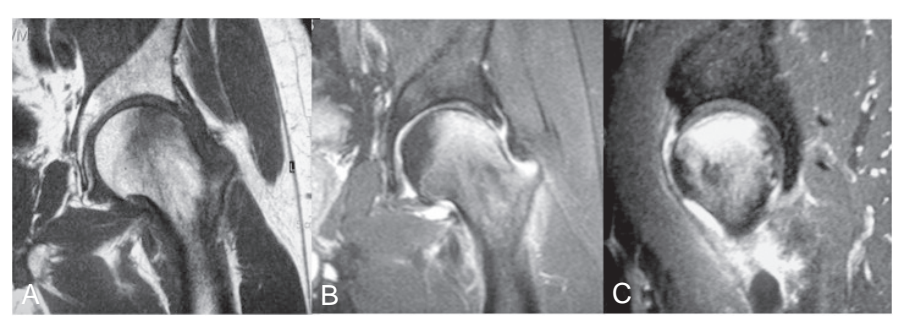

Figura 4 - MAF, masc. Fratura subcondral (seta preta) na porção ântero-superior da cabeça femoral esquerda. Caracteriza-se por traço linear com baixo sinal serpigiforme paralelo a superfície articular no plano coronal T1 (a), T2 com saturação de gordura no plano coronal (b) e sagital (c). Extenso edema da medular óssea $\left({ }^{*}\right)$ e derrame articular (seta branca).
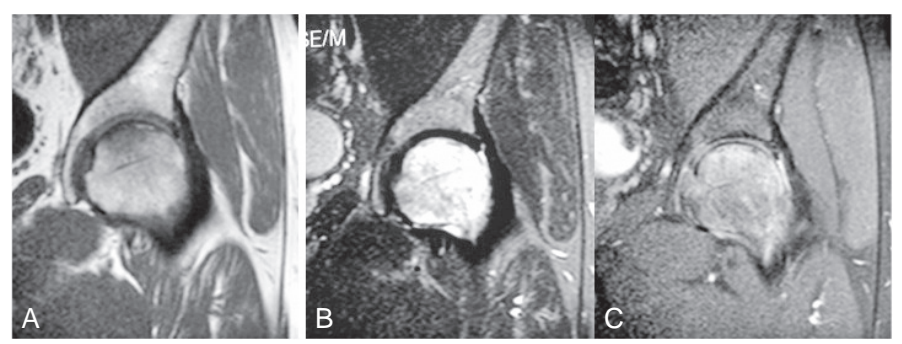

Figura 5 - MJC, masc, abril de 2007. Fratura subcondral (seta) na porção superior da cabeça femoral esquerda, associado a edema da medular óssea ( ${ }^{*}$ ). Plano coronal T1 (a), T2 com saturação de gordura (b) e T1 pós-contraste com saturação de gordura (c).

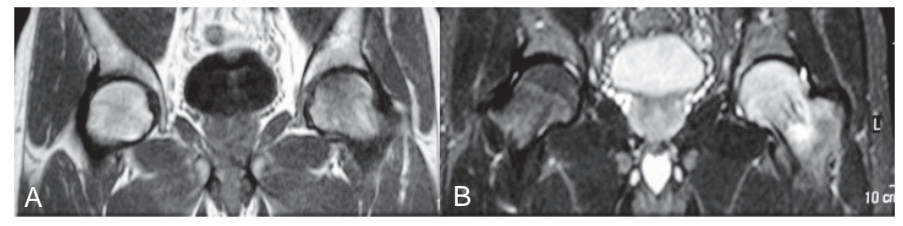

Figura 6 - MJC, masc, março de 2007. Fino traço de fratura subcondral (seta) melhor observada no plano coronal em T1 (a). Extenso edema da medular óssea $\left({ }^{*}\right)$ na cabeça femoral que se estende até a região intertrocantérica caracterizada por hipersinal em T2 com saturação de gordura (b). Não há sinais de colapso.

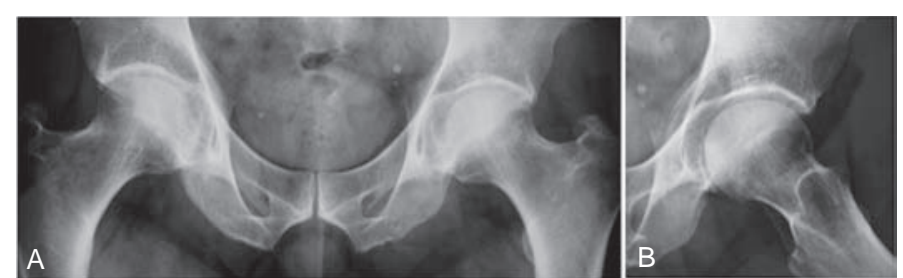

Figura 7 - ARL, masc, outubro de 2007. Estudo radiográfico de frente (a) e perfil (b). Radiografia de controle sem sinais de colapso ou osteonecrose. Espaço articular preservado.

A fratura subcondral caracteriza-se por fino traço de baixo sinal em todas as sequências, de localização subcondral, linear ou discretamente serpiginoso, paralelo à superfície articular ${ }^{(18)}$. Está associado a edema da medula óssea que se estende até o colo femoral.

\section{TRATAMENTO}

Vários tratamentos são possíveis nos casos de osteonecrose, desde conservador com medicação sintomática e alívio da carga até descompressão, osteotomia do fêmur proximal, enxertia com osso autógeno ou alógeno, enxertia de fíbula vascularizada com técnica microcirúrgica e artroplastia. O tratamento mais comumente utilizado para estágios precoces de osteonecrose é a descompressão da cabeça femoral, que objetiva diminuir a pressão intraóssea e normalizar o fluxo sanguíneo, levando à melhora da dor ${ }^{(4)}$.

É descrito que o tratamento conservador da osteoporose transitória, consistindo de anti-inflamatórios e alívio da carga, permite a resolução do quadro clínico e radiológico $^{(2,19)}$.

No período de janeiro de 2006 a dezembro de 2007 foram avaliados sete pacientes consecutivos com diagnóstico de fratura subcondral da cabeça femoral, atendidos pelo Grupo de Cirurgia do Quadril da Santa Casa de Misericórdia de São Paulo - Pavilhão "Fernandinho Simonsen”, com idade variando entre 37 e 60 anos (média de 50 anos). O sintoma principal era de dor na região inguinal, presente de duas a 16 semanas (média de oito semanas) antes da primeira consulta. Foram analisados 
seis pacientes do sexo masculino (86\%) e um do sexo feminino (14\%), sendo cinco quadris do lado esquerdo $(71,4 \%)$ e dois do direito $(28,6 \%)$. Nenhum dos pacientes relatou antecedente traumático grave.

Todos foram avaliados tanto clinicamente como com radiografias nas incidências de frente da bacia e perfil da articulação coxofemoral acometida e com ressonância magnética (RM) do quadril nos períodos pré e pós-tratamento. O controle com RM do quadril acometido foi realizado a cada quatro semanas para observar a melhora do edema medular.

Considerou-se como critério de melhora clínica a completa ausência de dor no quadril acometido e, como critério para liberação da carga no quadril afetado, a melhora total do edema medular demonstrado pela RM.

O tempo entre o início da dor e o diagnóstico de fratura subcondral variou de duas a 16 semanas (média de oito semanas). Em todos os casos, o tratamento foi realizado com alívio da carga no quadril com auxílio de muletas axilares e medicação sintomática de forma não padronizada por 24 a 30 semanas (média de 27,3 semanas). O tempo de seguimento variou de 13 a 20 meses (média de 15,5 meses). Houve resolução da dor de forma gradual por período que variou de nove a 10 meses (média de 9,7 meses) desde o início do quadro clínico. Nenhum paciente realizou descompressão. A resolução do edema medular foi observada nas RM de controle, realizadas a cada quatro semanas.

Neste estudo foram avaliados somente sete pacientes, porém, são casos consecutivos e essa entidade é rara.
Mais importante é que em todos esses casos foi realizado o diagnóstico diferencial e instituído o tratamento conservador. Ainda mais, sua real incidência pode ser maior, pelo diagnóstico equivocado de osteonecrose, tanto pelo ortopedista como pelo radiologista, em muitos casos de fratura subcondral.

O papel do radiologista é tão importante quanto o do ortopedista na diferenciação dessas afecções. Muito frequentemente, observamos o radiologista laudar essa afecção como osteonecrose avascular. Estabelecido o diagnóstico de osteonecrose, é importante definir a extensão da necrose, se há colapso da cabeça femoral e artrose secundária, enquanto que, definida a fratura subcondral, o mais importante é o alívio imediato da carga.

A confusão diagnóstica provoca erros de indicação de descompressão. Muitas vezes, casos de fratura subcondral são tratados como se fossem osteonecrose, melhorando sem colapso ou deformidades ${ }^{(5)}$. e são erroneamente computados como osteonecroses com boa evolução, fazendo muitos ortopedistas acreditarem que a descompressão é técnica salvadora. Enquanto não é feito o diagnóstico e promovido o alívio da carga nesses quadris, os pacientes não melhoram.

O correto diagnóstico da fratura subcondral da cabeça do fêmur evita medidas invasivas no quadril doente, que melhora de forma lenta, porém espontânea. O diagnóstico equivocado de osteonecrose da cabeça do fêmur pode ser evitado reconhecendo suas características e diferenças da fratura subcondral da cabeça do fêmur na ressonância magnética.

\section{REFERÊNCIAS}

1. Curtiss PH Jr, Kincaid WE. Transitory demineralization of the hip in pregnancy. A report of three cases. J Bone Joint Surg Am. 1959;41:1327-33.

2. Froberg PK, Braunstein EM, Buckwalter KA. Osteonecrosis, transient osteoporosis, and transient bone marrow edema: current concepts. Radiol Clin North Am. 1996;34(2):273-91.

3. Kim JW, Yoo JJ, Min BW, Hong SH, Kim HJ. Subchondral fracture of the femoral head in healthy adults. Clin Orthop Relat Res. 2007;(464):196-204.

4. Mitchell DG, Joseph PM, Fallon M, Hickey W, Kressel HY, Rao VM, et al. Chemical shift MR imaging of the femoral head: an in vitro study of normal hips and hips with avascular necrosis. AJR Am J Roentgenol. 1987;148(6):1159-64.

5. Mitchell DG, Rao VM, Dalinka MK, Spritzer CE, Alavi A, Steinberg ME, et al. Femoral head avascular necrosis: correlation of MR imaging, radiographic stanging, radionuclide imaging, and clinical findings. Radiology. 1987;162(3):709-15.

6. LiebermanJR, Berry DJ, Mont MA, Aaron RK, Callaghan JJ, Rajadhyaksha AD, et al. Osteonecrosis of the hip: management in the 21st century. Instr Course Lect. 2003;52:337-55.

7. Davies M, Cassar-Pullicino VN, Darby AJ. Subchondral insufficiency fractures of the femoral head. Eur Radiol. 2004;14(2):201-7.

8. Yamamoto T, Bullough PG. Subchondral insufficiency fracture of the femoral head. A differential diagnosis in acute onset of coxarthrosis in the elderly. Arthritis Rheum. 1999;42(12):2719-23.

9. Yamamoto T, Schneider R, Bullough PG. Subchondral insufficiency fracture of the femoral head: histopathologic correlation with MRI. Skeletal Radiol. 2001;30(5):247-54.

10. Hayes CW, Conway WF, Daniel WW. MR imaging of bone marrow edema pattern: transient osteoporosis, transient bone marrow edema syndrome, or osteonecrosis. Radiographics. 1993;13(5):1001-11.

11. Bloem JL. Transient osteoporosis of the hip: MR imaging. Radiology. 1988;167(3):753-5.

12. Turner DA, Templeton AC, Selzer PM, Rosenberg AG, Petasnick JP. Femoral capital osteonecrosis: MR finding of diffuse marrow abnormalities without focal lesions. Radiology. 1989;171(1):135-40.

13. Hofmann S, Engel A, Neuhold A, Leder K, Kramer J, Plenk H Jr. Bone-marrow oedema syndrome and transient osteoporosis of the hip. An MRI-controlled study of treatment by core decompression. J Bone Joint Surg Br. 1993;75(2):210-6.

14. Guerra JJ, Steinberg ME. Distinguishing transient osteoporosis from avascular necrosis of the hip. J Bone Joint Surg Am. 1995;77(4):616-24.

15. Rafii M, Mitnick H, Klug J, Firooznia H. Insufficiency fracture of the femoral head: MR imaging in three patients. AJR Am J Roentgenol. 1997;168(1):159-63.

16. Lafforgue P, Dahan E, Chagnaud C, Schiano A, Kasbarian M, Acquaviva PC. Early-stage avascular necrosis of the femoral head: MR imaging for prognosis in 31 cases with at least 2 years of follow-up. Radiology. 1993;187(1):199-204.

17. Yamamoto T, Schneider R. Bullough PG. Subchondral insufficiency fracture of the femoral head: histopathologic correlation with MRI. Skeletal Radiol. 2001;30(5): 247-54.

18. Takatori Y, Kokubo T, Ninomiya S, Nakamura S, Morimoto S, Kusaba I. Avascular necrosis of the femoral head. Natural history and magnetic resonance imaging. J Bone Joint Surg Br. 1993;75(2):217-21.

19. WilsonAJ, Murphy WA, Hardy DC, Totty WG. Transient osteoporosis: transient bone marrow edema? Radiology. 1988;167(3):757-60. 